\title{
CORRIGENDUM
}

\section{Molecular population genetics of the malaria vector Anopheles darlingi in central and South America}

\author{
L Mirabello and JE Conn
}

Heredity (2006) 97, 438. doi:10.1038/sj.hdy.6800913

Correction to: Heredity (2006) 96, 311-321. doi:10.1038/ sj.hdy.6800805

Since the publication of this issue, the authors have identified an error in the time to expansion estimate in the left-hand column of page 317. The South American calculation should have been 25312 years ago (95\% CI,
8555-40 242) not 253 119. The Amazonian and southern South American calculation should have been 16237 years ago (95\% CI, 5488-25814) not 162372.

The authors would like to apologize for any confusion caused. 